\title{
Barrelled Linearly Topologized Modules Over a Discrete Valuation Ring
}

\author{
Dinamérico P. Pombo Jr. ${ }^{1}$ \& Patricia Couto G. Mauro ${ }^{2}$ \\ ${ }^{1}$ Instituto de Matemática e Estatística, Universidade Federal Fluminense, Rio de Janeiro, Brasil \\ ${ }^{2}$ Centro Interdisciplinar de Ciências da Natureza, Universidade Federal da Integração Latino-Americana, Paraná, Brasil \\ Correspondence: Dinamérico P. Pombo Jr., Instituto de Matemática e Estatística, Universidade Federal Fluminense, Rua \\ Professor Marcos Waldemar de Freitas Reis, s/no., Bloco G, Campus do Gragoatá, 24210-201, Niterói, RJ, Brasil. E-mail: \\ dpombojr@gmail.com
}

\author{
Received: December 18, 2019 Accepted: January 13, 2020 Online Published: January 20, 2020 \\ doi:10.5539/jmr.v12n1p69 URL: https://doi.org/10.5539/jmr.v12n1p69
}

\begin{abstract}
In this paper barrelled linearly topologized modules over an arbitrary discrete valuation ring are introduced. A general form of the Banach-Steinhaus theorem for continuous linear mappings on barrelled linearly topologized modules is established and some consequences of it are derived.
\end{abstract}

Keywords: discrete valuation rings, linearly topologized modules, Banach-Steinhaus theorem

\section{Introduction}

Motivated by the fundamental works (Schwartz, 1950) (see also (Schwartz, 1966)) and (Dieudonné \& Schwartz, 1949), (Bourbaki, 1950) introduced the now classical barrelled locally convex spaces, which may be regarded as the Hausdorff locally convex spaces characterized by the validity of the Banach-Steinhaus property, and proved certain important facts related to that class of spaces (a quite complete study of such spaces may be found in (Pérez Carreras \& Bonet, 1987)).

In this paper the notion of a barrelled linearly topologized module over an arbitrary discrete valuation ring is introduced and it is shown that certain linearly topologized modules which are Baire spaces are barrelled. The main result established here is a general form of the Banach-Steinhaus theorem for continuous linear mappings on barrelled linearly topologized modules, from which the equicontinuity of separately equicontinuous sets of bilinear mappings between certain linearly topologized modules is derived. And, by using duality arguments, Hausdorff barrelled linearly topologized modules are characterized by the pertinent Banach-Steinhaus property. Additionally, known facts concerning modules of continuous linear mappings on certain linearly topologized modules which are Baire spaces are extended to the context of arbitrary barrelled linearly topologized modules.

A major difference between the classical case and the one discussed in the present work is that in the former invertible scalars (that is, non-zero scalars) may be taken "topologically close to zero", which does not occur in the context under consideration.

In this work $R$ will denote an arbitrary discrete valuation ring (Serre, 1968), unless otherwise specified, $M$ its valuation ring, $\pi$ a generator of $M$ and $R_{0}$ the $R$-module $K / R$ (where $K$ is the field of fractions of $R$ ); and " $R$-module" will always mean "unitary $R$-module". Since

$$
\left\{\pi^{n} R ; n=1,2, \ldots\right\}
$$

constitutes a fundamental system of neighborhoods of 0 in $R$ formed by ideals of $R$ such that $\bigcap_{n \geq 1} \pi^{n} R=\{0\}$, it follows that $R$ is a metrizable linearly topologized ring. Throughout this work we shall say that $E$ is a linearly topologized $R$-module if $E$ is a topological $R$-module whose origin admits a fundamental system of neighborhoods consisting of submodules of $E$. It is easily seen that a subset $B$ of a linearly topologized $R$-module $E$ is bounded if and only if for each neighborhood $U$ of 0 in $E$ which is a submodule of $E$ there is an integer $k \geq 1$ so that $\pi^{k} B \subset U$.

\section{Barrelled Linearly Topologized $R$-Modules: Basic Properties}

Definition 2.1. A subset $T$ of a linearly topologized R-module $E$ is said to be absorbing if it is a submodule of $E$ and for each $x \in E$ there exists an integer $l \geq 1$ so that $\pi^{l} x \in T$ (which is the same as $\left(\pi^{l} R\right) x \subset T$ ); $T$ is said to be a barrel in $E$ if $T$ is absorbing and closed in $E$. 
Remark 2.2. It is easily seen that the notion of an absorbing set does not depend on the choice of the generator $\pi$ of $M$.

Every neighborhood of 0 in a linearly topologized $R$-module $E$ which is a submodule of $E$ is a barrel in $E$. In the next example we shall see that the converse of this assertion is not always true.

Example 2.3. Assume that $R$ is complete. Let $E$ be the product $R$-module $R^{\mathbb{N}}$ endowed with the product topology, which makes $E$ into a metrizable and complete linearly topologized $R$-module, and let us consider the closed submodule $T=M^{\mathbb{N}}$ of $E$. Since it is clear that $\pi x \in T$ for all $x \in E, T$ is a barrel in $E$. On the other hand, it is clear that $T$ is not a neighborhood of 0 in $E$. We may also observe that, although $U=(\pi R) \times R \times R \times R \ldots$ is a neighborhood of 0 in $E$, $\pi U=\left(\pi^{2} R\right) \times(\pi R) \times(\pi R) \times(\pi R) \ldots$ is not a neighborhood of 0 in $E$.

Definition 2.4. A linearly topologized R-module $E$ is said to be barrelled if every barrel in $E$ is a neighborhood of 0 in $E$.

We have observed that the metrizable and complete linearly topologized $R$-module, considered in Example 2.3, is not barrelled.

Proposition 2.5. Let $E$ be a linearly topologized R-module such that $\pi^{n} U$ is a neighborhood of 0 in $E$ for each integer $n \geq 1$ and for each neighborhood $U$ of 0 in $E$. If $E$ is a Baire space, then $E$ is barrelled. In particular, if $E$ is metrizable and complete, then $E$ is barrelled.

Proof. Let $T$ be a barrel in $E$ and, for each integer $n \geq 1$, put

$$
U_{n}=\left\{x \in E ; \pi^{n} x \in T\right\} .
$$

Since $T$ is absorbing, $E=\bigcup_{n \geq 1} U_{n}$. Moreover, in view of the continuity of the $R$-linear mapping $\varphi_{n}: x \in E \mapsto \pi^{n} x \in E$ and of the closedness of $T$, it follows that $U_{n}=\varphi_{n}^{-1}(T)$ is closed. Therefore, since $E$ is a Baire space, there exists an integer $l \geq 1$ so that $\operatorname{int}\left(U_{l}\right) \neq \emptyset$. Let $y \in \operatorname{int}\left(U_{l}\right)$ and $U$ an open subset of $E$ such that $y \in U \subset U_{l}$. Then $V=U-U$ is a neighborhood of $0(=y-y)$ in $E$ such that $\pi^{l} V \subset T$. Consequently $T$ is a neighborhood of 0 in $E$ (since, by hypothesis, $\pi^{l} V$ is a neighborhood of 0 in $E$ ), and $E$ is barrelled.

As Example 2.3 shows, the condition " $\pi^{n} U$ is a neighborhood of 0 in $E$ for each integer $n \geq 1$ and for each neighborhood $U$ of 0 in $E$ " is essential for the validity of Proposition 2.5 .

Example 2.6. Assume that $R$ is complete. For each integer $n \geq 1$, the product $R$-module $R^{n}$ endowed with the product topology is a metrizable and complete linearly topologized R-module. Moreover, since

$$
\pi^{m}\left(\left(\pi^{m_{1}} R\right) \times \ldots \times\left(\pi^{m_{n}} R\right)\right)=\left(\pi^{m+m_{1}} R\right) \times \ldots \times\left(\pi^{m+m_{n}} R\right)
$$

for arbitrary integers $m, m_{1}, \ldots, m_{n} \geq 1$, Proposition 2.5 ensures that $R^{n}$ is barrelled.

Example 2.7. By Proposition 2.5, every discrete linearly topologized R-module is barrelled.

Proposition 2.8. Let $\left(E_{\alpha}\right)_{\alpha \in A}$ be a family of barrelled linearly topologized $R$-modules, $E$ an $R$-module and, for each $\alpha \in A$, let $u_{\alpha}$ be an R-linear mapping from $E_{\alpha}$ into $E$. Assume that $E=\left[\bigcup_{\alpha \in A} \operatorname{Im}\left(u_{\alpha}\right)\right]$ and that $E$ is endowed with the final linear topology for the family $\left(E_{\alpha}, u_{\alpha}\right)_{\alpha \in A}$ (Mauro \& Pombo Jr., 2015). Then the linearly topologized R-module E is barrelled.

Proof. Let $T$ be a barrel in $E$ and $\alpha \in A$ be arbitrary. We claim that the closed submodule $u_{\alpha}^{-1}(T)$ of $E_{\alpha}$ is a barrel in $E_{\alpha}$. In fact, let $x_{\alpha} \in E_{\alpha}$ be arbitrary. Since $T$ is a barrel in $E$, there is an integer $k \geq 1$ so that $\pi^{k} u_{\alpha}\left(x_{\alpha}\right) \in T$, that is, $\pi^{k} x_{\alpha} \in u_{\alpha}^{-1}(T)$. Hence, by the barrelledness of $E_{\alpha}, u_{\alpha}^{-1}(T)$ is a neighborhood of 0 in $E_{\alpha}$. Therefore $T$ is a neighborhood of 0 in $E$, proving that $E$ is barrelled.

An immediate consequence of Proposition 2.8 reads:

Corollary 2.9. (a) A quotient of a barrelled linearly topologized R-module, endowed with the quotient topology, is a barrelled linearly topologized $R$-module.

(b) A topological direct sum of a family of barrelled linearly topologized R-modules is a barrelled linearly topologized R-module.

Example 2.10. Assume that $R$ is complete. Then, in view of Example 2.6 and Corollary 2.9(b), the topological direct sum $R^{(\mathbb{N})}$ is barrelled. 


\section{The Banach-Steinhaus Theorem and Some Consequences}

If $E$ is a Hausdorff linearly topologized $R$-module, let $E^{*}$ be the $R$-module of all continuous $R$-linear mappings from $E$ into $R_{0}\left(R_{0}\right.$ endowed with the discrete topology, under which it is a barrelled linearly topologized R-module; recall Example 2.7) and let $\sigma\left(E, E^{*}\right)$ (resp. $\sigma\left(E^{*}, E\right)$ ) be the weak topology on $E$ (resp. $E^{*}$ ), which makes $E$ (resp. $E^{*}$ ) into a Hausdorff linearly topologized $R$-module (Mauro \& Pombo Jr., 2015).

Let us first establish a few simple results.

Proposition 3.1. If $T$ is an absorbing set in a Hausdorff linearly topologized R-module E, then

$$
T^{\perp}=\left\{\varphi \in E^{*} ; \varphi(t)=0 \text { for all } t \in T\right\}
$$

is $\sigma\left(E^{*}, E\right)$-bounded.

Proof. Let $x \in E$ be arbitrary and put

$$
U=\left\{\varphi \in E^{*} ; \varphi(x)=0\right\} .
$$

Since $T$ is absorbing, there is an integer $k \geq 1$ so that $\pi^{k} x \in T$. Thus

$$
\pi^{k} T^{\perp} \subset U,
$$

showing that $T^{\perp}$ is $\sigma\left(E^{*}, E\right)$-bounded.

Proposition 3.2. If $E$ is a Hausdorff linearly topologized $R$-module and $Y$ is a $\sigma\left(E^{*}, E\right)$-bounded subset of $E^{*}$, then

$$
Y^{\perp}=\{x \in E ; \varphi(x)=0 \text { for all } \varphi \in Y\}
$$

is a barrel in $E$.

Proof. By Proposition 4.11(a) of (Mauro \& Pombo Jr., 2015), $Y^{\perp}$ is a closed submodule of $E$. Let $x$ and $U$ be as in the proof of Proposition 3.1. By the $\sigma\left(E^{*}, E\right)$-boundedness of $Y$ there is an integer $l \geq 1$ so that $\pi^{l} Y \subset U$. Consequently, $\varphi\left(\pi^{l} x\right)=0$ for all $\varphi \in Y$, that is, $\pi^{l} x \in Y^{\perp}$. Therefore $Y^{\perp}$ is a barrel in $E$.

Proposition 3.3. For a subset $T$ of a Hausdorff linearly topologized R-module $E$, the following conditions are equivalent:

(a) $T$ is a barrel in $E$;

(b) there exists a $\sigma\left(E^{*}, E\right)$-bounded subset $Y$ of $E^{*}$ such that $T=Y^{\perp}$.

Proof. (a) $\Rightarrow$ (b): Since $T$ is a closed submodule of $E$, Proposition 4.12 of (Mauro \& Pombo Jr., 2015) furnishes $T=$ $T^{\perp \perp}=\left(T^{\perp}\right)^{\perp}$. But, since $T$ is absorbing, Proposition 3.1 guarantees that $T^{\perp}$ is $\sigma\left(E^{*}, E\right)$-bounded. Thus it is enough to take $Y=T^{\perp}$

(b) $\Rightarrow$ (a): Follows immediately from Proposition 3.2.

If $E$ and $F$ are linearly topologized $R$-modules, $\mathcal{L}(E, F)$ will denote the $R$-module of all continuous $R$-linear mappings from $E$ into $F$ and $\tau_{s}$ the topology of simple convergence on $\mathcal{L}(E, F)$, under which $\mathcal{L}(E, F)$ is a linearly topologized $R$-module (which is a Hausdorff space if $F$ is a Hausdorff space).

Our main result is the following version of the Banach-Steinhaus theorem:

Theorem 3.4. If $E$ is a barrelled linearly topologized $R$-module and $F$ is an arbitrary linearly topologized R-module, then every $\tau_{s}$-bounded subset $\mathcal{X}$ of $\mathcal{L}(E, F)$ is equicontinuous.

Proof. Let $V$ be a neighborhood of 0 in $F$ which is a submodule of $F$ (then $V$ is closed in $F$ ) and put

$$
U=\bigcap_{u \in \mathcal{X}} u^{-1}(V) \text {. }
$$

We claim that the closed submodule $U$ of $E$ is a barrel in $E$. Indeed, if $x \in E$ is arbitrary, the boundedness of $X(x)=$ $\{u(x) ; u \in \mathcal{X}\}$ guarantees the existence of an integer $k \geq 1$ so that $\pi^{k} \mathcal{X}(x) \subset V$. Thus $\pi^{k} x \in u^{-1}(V)$ for all $u \in \mathcal{X}$, that is, $\pi^{k} x \in U$. Therefore $U$ is a barrel in $E$ and the assumption that $E$ is barrelled implies that $U$ is a neighborhood of 0 in $E$. Consequently $\mathcal{X}$ is equicontinuous, as was to be shown.

We have the following characterization of Hausdorff barrelled linearly topologized $R$-modules by means of the BanachSteinhaus property: 
Proposition 3.5. For a Hausdorff linearly topologized R-module E, the following conditions are equivalent:

(a) E is barrelled;

(b) for every Hausdorff linearly topologized $R$-module $F$, we have that each $\tau_{s}$-bounded subset of $\mathcal{L}(E, F)$ is equicontinuous;

(c) each $\sigma\left(E^{*}, E\right)$-bounded subset of $E^{*}$ is equicontinuous.

Proof. Since the implication (a) $\Rightarrow(b)$ is a particular case of Theorem 3.4 and the implication (b) $\Rightarrow(c)$ is obvious, it remains to prove that $(\mathrm{c}) \Rightarrow(\mathrm{a})$. Indeed, let $T$ be a barrel in $E$. By Proposition 3.3 there is a $\sigma\left(E^{*}, E\right)$-bounded subset $Y$ of $E^{*}$ such that $T=Y^{\perp}$; and, by (c), $Y$ is equicontinuous. Hence there exists a neighborhood $U$ of 0 in $E$ such that $Y(U)=\{0\}$, which implies $U \subset Y^{\perp}=T$. Thus $T$ is a neighborhood of 0 in $E$, proving (a).

Corollary 3.6. Let $E$ be a barrelled linearly topologized $R$-module and $F$ a Hausdorff linearly topologized $R$-module. If $\left(u_{i}\right)_{i \in I}$ is a net in $\mathcal{L}(E, F)$ which is $\tau_{s}$-bounded and pointwise convergent to a mapping $u: E \rightarrow F$, then $u \in \mathcal{L}(E, F)$. In particular, if $\left(u_{n}\right)_{n \geq 1}$ is a sequence in $\mathcal{L}(E, F)$ pointwise convergent to a mapping $u: E \rightarrow F$, then $u \in \mathcal{L}(E, F)$.

Proof. Since $F$ is a Hausdorff space, it is easily seen that $u$ is an $R$-linear mapping. And, by Theorem 3.4, the set $\mathcal{X}=\left\{u_{i} ; i \in I\right\}$ is equicontinuous. Consequently, $u \in \mathcal{L}(E, F)$, as asserted.

Corollary 3.7. Let $E, F$ be linearly topologized R-modules, E being barrelled and $F$ quasi-complete and a Hausdorff space. If $\mathcal{B}$ is a covering of $E$ by bounded subsets of $E$ which is stable under finite unions, then $\left(\mathcal{L}(E, F), \tau_{\mathcal{B}}\right)$ is quasicomplete, where $\tau_{\mathcal{B}}$ is the (Hausdorff linear $R$-module) topology of $\mathcal{B}$-convergence on $\mathcal{L}(E, F)$.

Proof. Let $\mathcal{X}$ be a $\tau_{\mathcal{B}}$-closed and $\tau_{\mathcal{B}}$-bounded subset of $\mathcal{L}(E, F)$. Then $\mathcal{X}$ is $\tau_{s}$-bounded, and Theorem 3.4 ensures its equicontinuity. By Proposition 3.6 of (Mauro \& Pombo Jr., 2015), $\mathcal{X}$ is $\tau_{\mathcal{B}}$-complete. Therefore $\left(\mathcal{L}(E, F), \tau_{\mathcal{B}}\right)$ is quasicomplete.

Let $R$ be arbitrary and consider the submodule $E=R^{(\mathbb{N})}$ of $R^{\mathbb{N}}$ endowed with the topology induced by the product topology on $R^{\mathbb{N}}$. By arguing as in Example 2.3 one sees that the metrizable linearly topologized $R$-module $E$ is not barrelled. We have seen in Example 3.15 of (Mauro \& Pombo Jr., 2015) that the separately continuous $R$-bilinear mapping

$$
\left(\left(\lambda_{n}\right)_{n \geq 1},\left(\mu_{n}\right)_{n \geq 1}\right) \in E \times E \longmapsto \sum_{n=1}^{\infty} \lambda_{n} \mu_{n} \in R
$$

is not continuous. In the next proposition we shall prove that every separately continuous $R$-bilinear mapping on a product of two metrizable linearly topologized $R$-modules, one of them being barrelled, is necessarily continuous. But, before stating the promised result, let us recall that if $E, F$ and $G$ are arbitrary linearly topologized $R$-modules and $\mathcal{X}$ is a separately equicontinuous set of $R$-bilinear mappings from $E \times F$ into $G$ which is equicontinuous at $(0,0)$, then $\mathcal{X}$ is equicontinuous. In fact, for $x, h \in E, y, k \in F$ and $u \in \mathcal{X}$, one has

$$
u(x+h, y+k)-u(x, y)=u(h, k)+u(x, k)+u(h, y) .
$$

We shall establish the following result, which was motivated by Theorem 3, p. 28 of (Bourbaki, 1967).

Proposition 3.8. Let $E, F$ be metrizable linearly topologized R-modules, with E barrelled, and let $G$ be an arbitrary linearly topologized $R$-module. If $\mathcal{X}$ is a separately equicontinuous set of $R$-bilinear mappings from $E \times F$ into $G$, then $\mathcal{X}$ is equicontinuous.

Proof. In view of what we have just mentioned, it suffices to show that, for each null sequence $\left(x_{n}\right)_{n \geq 1}$ in $E$ (resp. ( $\left.y_{n}\right)_{n \geq 1}$ in $F),\left(u\left(x_{n}, y_{n}\right)\right)_{n \geq 1}$ converges uniformly to 0 for $u \in \mathcal{X}$. In order to reach our purpose, we first claim that the set

$$
\mathcal{Y}=\left\{x \in E \longmapsto u\left(x, y_{n}\right) \in G ; u \in \mathcal{X}, n=1,2, \ldots\right\}
$$

is equicontinuous. But, since $E$ is barrelled, it is enough to show that $\mathcal{Y}$ is $\tau_{s}$-bounded in $\mathcal{L}(E, G)$ (Theorem 3.4). In fact, if $x \in E$ is arbitrary, the equicontinuity of the set

$$
\{y \in F \longmapsto u(x, y) \in G ; u \in \mathcal{X}\}
$$

and the boundedness of the set $\left\{y_{n} ; n=1,2, \ldots\right\}$ imply the boundedness of the set $\mathcal{Y}(x)=\left\{u\left(x, y_{n}\right) ; u \in \mathcal{X}, n=1,2, \ldots\right\}$. Therefore $\mathcal{Y}$ is equicontinuous. Consequently, given an arbitrary neighborhood $V$ of 0 in $G$, there exists a neighborhood 
$U$ of 0 in $E$ such that $\mathcal{Y}(U) \subset V$. Since $\left(x_{n}\right)_{n \geq 1}$ is a null sequence in $E$, there exists an integer $m \geq 1$ so that $x_{n} \in U$ for $n \geq m$, and hence $u\left(x_{n}, y_{n}\right) \in V$ for $n \geq m$ and $u \in \mathcal{X}$. Thus $\left(u\left(x_{n}, y_{n}\right)\right)_{n \geq 1}$ converges uniformly to 0 for $u \in \mathcal{X}$, and the proof is concluded.

Finally we would like to mention that a special case of Theorem 3.4 (resp. Corollary 3.6, Corollary 3.7, Proposition 3.8) has been obtained in (Mauro \& Pombo Jr., 2015).

\section{References}

Bourbaki, N. (1950). Sur certains espaces vectoriels topologiques. Ann. Inst. Fourier, 2, 5-16. https://doi.org/10.5802/aif.16

Bourbaki, N. (1967). Espaces Vectoriels Topologiques, chapitres 3 à 5, Actualités Scientifiques et Industrielles 1229. Hermann, Paris.

Dieudonné, J., \& Schwartz, L. (1949). La dualité dans les espaces $\mathcal{F}$ et $\mathcal{L F}$. Ann. Inst. Fourier, 1, 61-101. https://doi.org/10.5802/aif.8

Mauro, P. C. G., \& Pombo, D. P. (2015). Linearly topologized modules over a discrete valuation ring. Boll. Unione Mat. Ital., 7 , 253-278. https://doi.org/10.1007/s40574-014-0015-8

Pérez, C. P., \& Bonet, J. (1987). Barrelled Locally Convex Spaces, Notas de Matemática 113. North-Holland, Amsterdam.

Schwartz, L. (1950). Théorie des Distributions, tome 1, Actualités Scientifiques et Industrielles 1091. Hermann, Paris.

Schwartz, L. (1966). Théorie des Distributions. Hermann, Paris.

Serre, J.-P. (1968). Corps Locaux, Quatrième édition, Actualités Scientifiques et Industrielles 1296. Hermann, Paris.

Warner, S. (1989). Topological Fields, Notas de Matemática 126. North-Holland, Amsterdam.

\section{Copyrights}

Copyright for this article is retained by the author(s), with first publication rights granted to the journal.

This is an open-access article distributed under the terms and conditions of the Creative Commons Attribution license (http://creativecommons.org/licenses/by/4.0/). 